\title{
Reliability Prediction in Electronic Packages Using Molecular Simulation
}

\author{
H. B. FAN, Cell K.Y. Wong, Matthew M.F. YUEN \\ Department of Mechanical Engineering, \\ Hong Kong University of Science and Technology \\ Clear Water Bay, Kowloon, Hong Kong SAR, China \\ Email:mehaibo@ust.hk, \\ Phone: (852) 2358 8814; FAX: (852) 23581202
}

\begin{abstract}
Reliability of electronic packages is a great concern to packaging design engineers. During its design life, packages experience a wide range of temperature variations. The mismatch in coefficient of thermal expansion between the different layers in the packages can generate high interfacial stresses due to these thermal loading. If these stresses exceed the limiting value, delamination will occur.

The present study is focused on the reliability of the epoxy molding compound (EMC) and cuprous oxide coated copper substrate. In order to verify whether the interfacial adhesion is dominant by the content of cuprous oxide on the cooper substrate, two models were built to simulate the thermal cycling test with a constant cuprous oxide and a changing content of cuprous oxide on the copper substrate. The thermal cycling test was conducted with a given thermal profile. The adhesion strength between EMC and cuprous oxide coated copper substrate at different thermal cycles was evaluated using the button shear test (BST). A simple molecular model of a bi-material system, which consists of EMC and cuprous oxide coated copper substrate, was built to evaluate the interfacial energy of the Cu-EMC system. In order to dramatically reduce the computational time, the system was modeled with a limited number of atoms. A preset strain was applied to the model representing a forcing step as the EMC material was pulled away from the copper substrate. Equilibration was conducted to relax the whole system before the next strain step proceeded. The procedure was repeated using different strains. The interfacial energy at different thermal cycles was evaluated. The variation of the interfacial energy indicated the change in the adhesion strength between EMC and cuprous oxide coated copper during the thermal cycling test. The simulation results revealed that the cuprous oxide content in the copper substrate had a large effect on the adhesion between EMC and copper, which is consistent with the experimental observation.
\end{abstract}

\section{Introduction}

Recently electronic package design is moving towards not only high speed and multi-functional application, but also high density packaging with high performance requirements. The mismatch in the coefficient of thermal expansion between different layers in packages can generate high interfacial stresses when subjected to thermal loading during fabrication and assembly. These stresses concentrate at the locations of geometric discontinuities and often cause delaminations. Reliability analysis is needed in order to ensure the electronic packages meeting the demanding performance requirements.
Fan et al [1] used finite element analysis to investigate delamination in flip chip package under thermal cycling test and Basaran et al. [2] studied the reliability of the package subjected thermal cycling loading. Although the method can help to understand the failure mechanism in reliability tests, some phenomena in the tests still cannot be completely explained using the conventional fracture mechanics theory. Moreover, as material properties at the interface are different from those of bulk materials, such as, the effect of copper oxide on the adhesion between EMC and copper, it is important to better understand reliability at a molecular level.

Molecular modeling represents molecular structures numerically and simulates their behavior with the equations of quantum and classical physics and it is one of the fastest growing fields in science. The molecular dynamics (MD) method was first introduced by Alder and Wainwright in the late 1950's to study the interactions of hard spheres [3]-[4]. Yarovsky [5] used MD simulations to investigate the fundamentals of adhesion at various interfaces of the coating system and revealed the molecular mechanisms of the adhesion formulation and failure. Property trend analysis and simulation of the adhesive formulation effect in the microelectronics packaging industry using MD simulation were conducted by Iwamoto [6][7], and she evaluated the performace of electronic package using MD simulations [8][9]. MD simulations were also conducted to investigate the molecular interactions between different nano structures and interesting results were presented by other researchers [1012].

In this study, MD simulation was conducted to investigate the adhesion in a Cu-EMC system under thermal cycling loading on a molecular level. Two MD models, one with various and the other with constant cuprous oxide content on the copper substrate, were built. The MD simulation results explained the some of the phenomena observed in the thermal cycling test, and it indicated a basic relation between the molecular performance and the macroscopic performance. The models revealed that the cuprous oxide content of the copper substrate had a large effect on the adhesion strength between the EMC and copper, which is consistent with the experimental observation.

\section{Simulation of the Thermal Cycling Test}

Thermal cycling test were conducted and the results from the button shear test to measure the varying of the adhesion strength at various stages of the thermal cycling test were presented by Chung, et al [13]. A similar trend was obtained among all batches of samples, showing an increase in adhesion strength within the range of the first 500 to 700 thermal cycles, as shown in Figure 1. After reaching the peak, 
degradation of interfacial adhesion strength was observed. The adhesion strength decreased subsequently in its rundown to 2000 thermal cycles. In order to explain this phenomenon, Chung et al measured the nature and the content of copper oxide on the copper substrate surface subjected to thermal cycling loading using the X-ray Photoelectron Spectroscopy (XPS), and found that there was only cuprous oxide for all the sheared samples. She also found that the content of cuprous oxide was increased from 14 to 21 atomic concentrations during the 0 to about 600 thermal cycles range. Then the value of the cuprous oxide content was reduced to around 15 for the rest of the thermal cycles. The changing trend of cuprous oxide content is similar to that of the adhesion force versus number of thermal cycles.

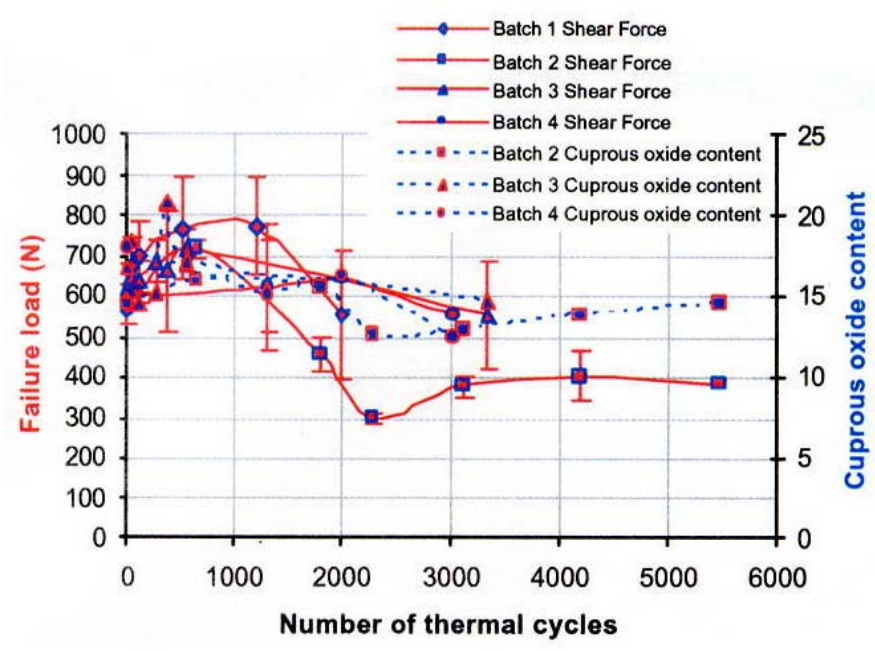

Figure 1: The adhesion force and cuprous oxide content against different numbers of thermal cycles (Chung et al)

Based on Chung's results, a layer of cuprous oxide was introduced onto the copper surface in the MD model as shown in Figure 2. The model consisting of the EMC and copper substrate coated with cuprous oxide was built within a rectangular simulation cell of $14.43 \times 20.42 A^{2}$ in the $\mathrm{x}$ and $\mathrm{y}$ directions, periodic in the plane parallel to the surface. All the atoms of copper and cuprous oxide were held rigid, while all the atoms of EMC were allowed to move freely. According to the molding condition, the simulation was performed at a temperature of $175^{\circ} \mathrm{C}$ with a pressure of 4.3Mpa, using the constant-pressure and temperature ensemble (NPT). Then the whole system was equilibrated for about $100 \mathrm{ps}$ at a temperature of $125^{\circ} \mathrm{C}$ using the ensembles of the constant number of particles, constant-volume and constant temperature (NVT), which was followed by a cooling simulation from $125^{\circ} \mathrm{C}$ to $25^{\circ} \mathrm{C}$ using NVT. The above simulation procedure reproduces the whole fabrication steps of the button shear test samples.

In the molecular dynamics simulations, a periodic model of the $\mathrm{EMC} / \mathrm{Cu}$ system was used. By applying periodic boundaries to the simulations, the influence of bulk environments could be included, therefore improving the accuracy and realism of the model. Hence, the properties of a molecular level system and a macroscopic system are essentially the same. In this study, the periodic model of the bi-material system included a fragment of EMC and copper atoms.

The condensed-phase optimized molecular potential for atomistic simulation studies (COMPASS) force field was used in the simulation. COMPASS is an ab initio force field that enables accurate and simultaneous prediction of structural, conformational, vibrational, and thermo-physical properties for a broad range molecules in isolation and in condense phases under a wide range of conditions of temperature and pressure. The advantage of the COMPASS force field is of its high accuracy in predicting the molecular properties of polymers, metals and their interfaces.

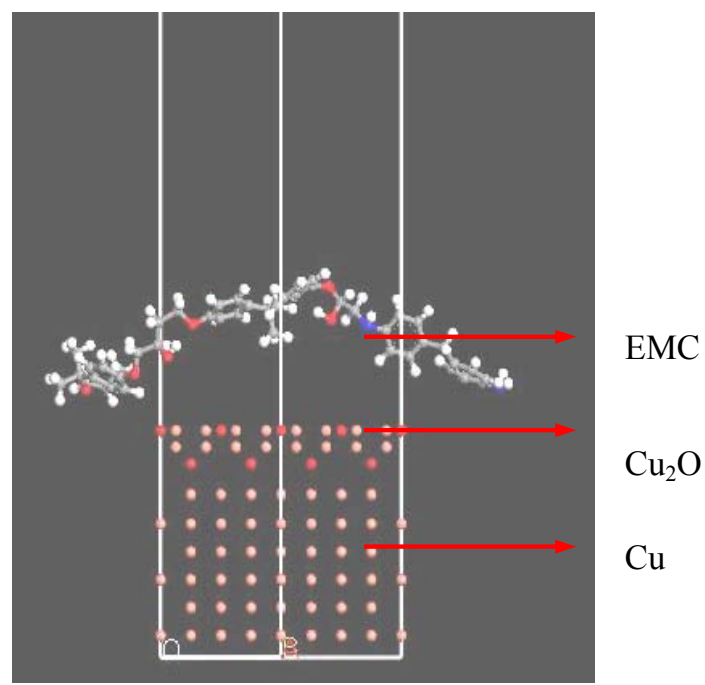

Figure 2: The reliability model of the EMC and copper system

In order to reproduce the behaviour of adhesion between the EMC and copper substrate in the thermal cycling test, the amount of cuprous oxide atoms in the model changed with thermal cycles. In this simulation we assumed that there was an initial amount of cuprous oxide atoms on the copper surface at zero cycle, and it changed with thermal cycles at the same rate as that of experiments conducted by Chung, et al [13].

The thermal cycling model was constructed using a preset strain target during each simulation step. In a cooling step, a set strain was applied to the model during a forcing step in which the EMC material was pulled away from the copper substrate. Equilibration was conducted to relax the whole system at the cooling temperature; while in a heating step, a different strain was applied to the model during a forcing step in which the EMC material was pushed close to the copper substrate. The whole system was then relaxed at the heating temperature. The cooling and heating procedure was repeated using different strains with different amounts of cuprous oxide atoms.

\section{Results and Discussion}

Figure 3 shows the molecular structure of the EMC and copper system under thermal cycling test at zero cycle and at 1800 thermal cycles respectively. It can easily be seen that 
the configuration of EMC after 1800 cyclesis different from its initial form, and a large void exists at the interface after thermal cycling, which will reduce the adhesion between the EMC and the copper substrate. The reason is that the interfacial stresses resulting from the CTE mismatch of EMC and copper pull the EMC away from the copper substrate step by step during the thermal cycling. Therefore, increasing number of voids will form cracks at the interface which makes the interface weaker after 1800 thermal cycles.

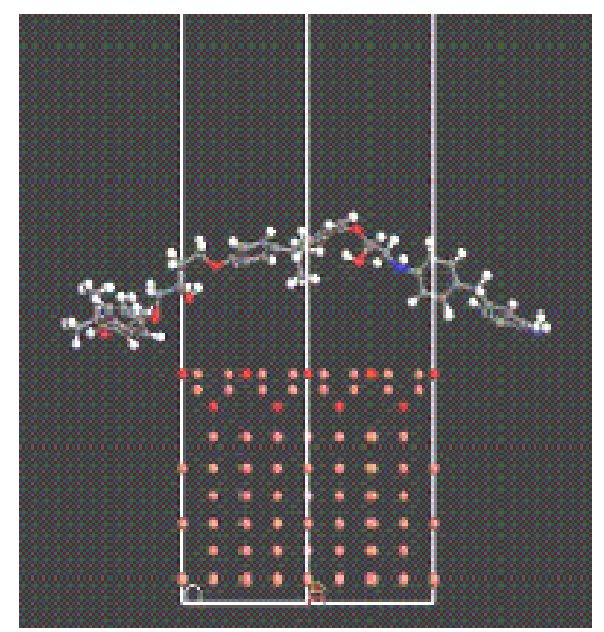

(a)

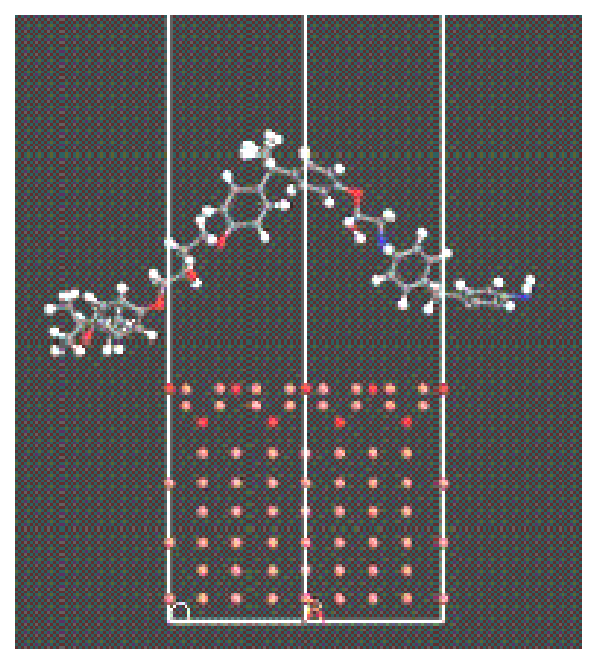

(b)

Figure 3: MD simulation thermal cycling (a) 0 cycle (b)1800 cycles

The bonding strength between the EMC and the copper substrate can be evaluated by examining the potential energy. The total potential energy was represented by the superposition of valence and non-bond interactions. The valence terms consist of bond stretch, bond angle bending, and dihedral angle torsion terms, while non-bond interactions consist of van der Waals and electrostatic terms. The interaction between the EMC and the copper tip is a non-bond interaction. Hence the interfacial bonding energy came from the electrostatic and van der Waals forces in the molecular system. Generally, the interaction energy was estimated from the energy difference, $\Delta E$, between the total energy of the whole system and the sum of the energies of individual molecules as follows:

$$
\Delta E=E_{\text {total }}-\left(E_{E M C}+E_{\mathrm{Cu}+\mathrm{Cu}_{2} \mathrm{O}}\right)
$$

where $E_{\text {total }}$ is the total energy of the whole system, $E_{E M C}$ is the energy of the EMC without the copper substrate, $E_{\mathrm{Cu}+\mathrm{Cu}_{2} \mathrm{O}}$ is the energy of the copper substrate without the EMC. The interfacial bonding energy, $\gamma$, was evaluated using the interfacial energy $\Delta E$ and the contact area $A$ between the copper and EMC:

$$
\gamma=\Delta E / A
$$

Based on above equations, the bonding energies between the EMC and copper substrate with cuprous oxide were plotted against thermal cycles, as shown in Figure 4. The bonding energy increased within the first 600 thermal cycles and reached the maximum value at about 600 thermal cycles, and then the bonding energy decreased for the remainder of the thermal cycles. This curve showed a similar trend as those of cuprous oxide content and adhesion force in the thermal cycling test. The higher bonding energy means that higher the force is needed to separate the EMC from the copper substrate. Therefore the result is consistent with the phenomena in the thermal cycling test on the molecular level.

In order to verify whether the interfacial adhesion is dominant by the content of cuprous oxide on the cooper substrate, another MD model was built for the thermal cycling with a constant content of cuprous oxide on the copper substrate. Based on the same procedure as addressed in the above simulation, the constant cuprous oxide simulations were conducted and the corresponding interfacial bonding energy at different stages of the thermal cycles was calculated and also plotted in Figure 4. It can be seen that the bonding energy for the thermal cycling test with a constant cuprous oxide content generally decreased with the increase of the thermal cycle. The comparison of the result from the two kinds of simulations indicates that the phenomena in the thermal cycling test mainly results from the change of the cuprous oxide content on the copper substrate. Higher content of the cuprous oxide on the copper substrate results in higher the adhesion strength between EMC and copper substrate, which is demonstrated by the result of Chung et al [13]. It is also shown by $\mathrm{Su}$ [14] that the cuprous oxide is the key ingredient for better adhesion by detecting a higher adhesion for the cuprous oxide on the copper wire.

This MD result indicates that the adhesion strength of the EMC/copper interface, while being affected by the bonding material system, is more significantly affected by the chemical composition of the material system at the interface. This is consistent with the observation in the thermal cycling test result. Study of surface adhesion by Kendall [15] has also suggested that the adhesion between surfaces is governed by a number of factors such as Van der Waal force, linking of the polymer chain, surface cleanliness and roughness. He argued that the bonding between adhesive and substrate surface 


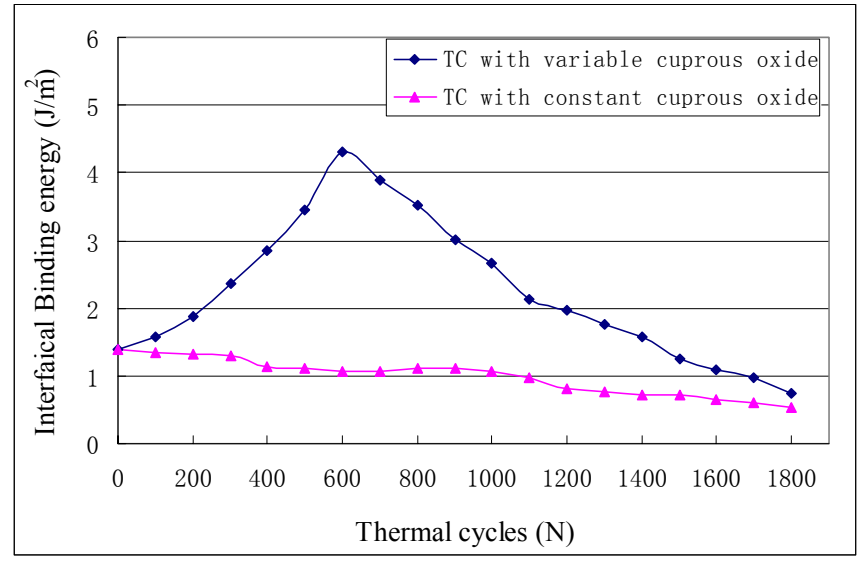

Figure 4: The layer element at the interface (TC: Thermal cycling)

depends on the surface energy. This strongly related to the surface properties of the bonding surface rather than the bulk material properties of the two bonded materials.

The results from MD simulations demonstrated interactions between EMC and copper oxide substrate during the thermal cycling test. The basic trend of adhesion followed an interaction energy trend. Higher interaction energy results in higher adhesion strength between EMC and cuprous oxide coated copper. Although the values from MD simulations could not be quantitatively compared to those of the experimental measurements, qualitative trends predicted by MD simulation have shown to be in agreement with experimental observations. And the simulation results can be used as a guide for further researches.

\section{Conclusions}

The paper is focused on reliability prediction using a computational model during the thermal cycling test. A computational model was developed to investigate the adhesion of the EMC-copper oxide system during the thermal cycling test. The present molecular model helped to explain results in the thermal cycling test, and it indicates a basic relation between the molecular performance and the macroscopic performance. The model revealed that the cuprous oxide content in the copper substrate had a large effect on the adhesion between EMC and copper. Higher content of surface cuprous oxide on the copper substrate gives higher adhesion strength between EMC and copper substrate, which is consistent with the experimental observation. It is also revealed that the adhesion was dominant by the interfacial material properties, rather than the bulk material properties.

\section{Acknowledgments}

The project was supported by the Research Grant Council project (HKUST6046/01E). The authors would like to thank Dr. Iwamoto for her suggestion on the study.

\section{References}

[1] X. J. Fan, H. B. Wang and T. B. Lim, “ Investigation of the Underfill Delamination and Cracking in Flip-chip Modules under Temperature Cyclic Loading," IEEE
Electronic Components and Technology Conf. pp. 9941000, 1994.

[2] C. Basaran, R. Chandaroy, "Finite Element Simulation of the Temperature Cycling Tests," Components, Packaging and Manufacturing Technology, Part A, IEEE Transactions, Vol. 20, pp. 530-536, 1997.

[3] B. J. Alder, T. E. Wainwright, "Phase Transition for a Hard Sphere System, Journal of Chemical Physics, Vol. 27, pp. 1208-1211, 1957.

[4] B. J. Alder, T. E. Wainwright, "Studies in Molecular Dynamics. I. General Method," Journal of Chemical Physics, Vol. 31, pp. 459-466, 1959.

[5] I. Yarovsky, "Atomic Simulation of Interface in Materials: Theory and Applications," Aust. J. Phys., Vol.503, pp. 407-424, 1997.

[6] N. Iwamoto, J. Pedigo, "Property Trend Analysis and Simulations of Adhesiove Formulation Effects in the Microelectronics Packaging Industry Using Molecular Modeling," 48th Electron. Comp. Tech. Conf., USA, pp. 1241-1246, 1998.

[7] N. Iwamoto, "Applying Polymer Process Studies Using Molecular Modelling," Adhesive Joining and Coating Technology in Electronics Manufacturing, 4th International Conference, pp. 182-187, 2000.

[8] N. Iwamoto, "Advancing Materials Using Interfacial Process and Reliability Simulations on The Molecular Level," Advanced Packaging Materials: Processes, Properties and Interfaces, pp. 14-17, 2000.

[9] N. Iwamoto, “Advancing Polymer Process Understanding in package and Borard Applications through Molecular Modeling," 50th Electron. Comp. Tech. Conf., USA, pp. 1354-1359, 2000.

[10] Z. Y. Liang, J. Gou, C. Zhang, B. Wang, L. Kramer, and, "Investigation of Molecular Interactions between $(10,10)$ Single -walled Nanotube and Epon 962 Resin/DETDA Curing Agent Molecules," Materials Science and Engineering, A365, pp.228-234, 2004.

[11] J. Mijovie, and H. Zhang, "Molecular Dynamic Simulation of Study of Motions and Interactions of Water in a Polymer network," J. Phys. Chem. B, vol. 108, pp. 2557-2563, 2004.

[12] S. J. V. Frankland, A. Caglar, D. W. Brenner, and M. Griebel, " Molecular Simulation of the Influence of Chemical Cross-links on the Shear Strength of Carbon Nanotube -polymer Interfaces," J. Phys. Chem. B, vol. 106, pp. 3046-3048, 2002.

[13] P. W. Chung, M.M.F Yuen, P. C. H. Chan, N. K. C. Ho and D.C.C. Lam, "Effect of Copper Oxide on the Adhesion behaviour of Epoxy Moulding Compoundcopper Interface," 52nd Electronic Packaging Technology Conference, 2002, pp.1665-1670.

[14] Y. Y. Su, and R. M. Shemenski, "The Role of Oxide Structure on Copper Wire to the Rubber Adhesion," Applied Surface Science, Vol. 161(3-4), pp. 355364,2000 .

[15] K. Kendall, "Molecular adhesion and its applications: the sticky universe", Kluwer Academic / Plenum Publishers, 2001. 